\title{
Fabrication of bulk nanocomposite magnets via severe plastic deformation and warm compaction
}

\author{
Chuanbing Rong, ${ }^{1}$ Ying Zhang, ${ }^{1,2}$ Narayan Poudyal, ${ }^{1}$ Xiangyuan Xiong, ${ }^{3}$ M. J. Kramer, ${ }^{2}$ \\ and J. Ping $\mathrm{Liu}^{1, \text { a) }}$ \\ ${ }^{1}$ Department of Physics, University of Texas at Arlington, Arlington, Texas 76019, USA \\ ${ }^{2}$ Division of Materials Science and Engineering, Ames Laboratory, Iowa State University, \\ Ames, Iowa 50011, USA \\ ${ }^{3}$ Department of Materials Engineering, Monash Center for Electron Microscopy, Monash University, \\ Clayton, Victoria 3800, Australia
}

(Received 23 November 2009; accepted 16 February 2010; published online 12 March 2010)

\begin{abstract}
We demonstrate that a $\mathrm{SmCo} / \mathrm{FeCo}$ based hard/soft nanocomposite material can be fabricated by distributing the soft magnetic $\alpha$-Fe phase particles homogeneously in a hard magnetic SmCo phase through severe plastic deformation. The soft-phase particle size can be reduced from micrometers to smaller than $15 \mathrm{~nm}$ upon deformation. Up to $30 \%$ of the soft phase can be incorporated into the composites without coarsening. A warm compaction process of the plastically deformed powder particles then produces bulk nanocomposite magnets of fully dense nanocomposites with energy product up to 19.2 MGOe owing to effective interphase exchange coupling, which makes this type of nanocomposite magnets suitable for high energy-density applications at high temperatures. (C) 2010 American Institute of Physics. [doi:10.1063/1.3358390]
\end{abstract}

Exchange-coupled nanoscale composite magnets consisting of a hard magnetic phase that provides high coercivity and a soft magnetic phase that provides high magnetization have potential to be the next generation of permanent magnets with very high energy products. ${ }^{1,2}$ Experimental efforts in the past two decades have demonstrated that energy product enhancement can be achieved in low dimensional hard/ soft magnetic systems including physically deposited thin films ${ }^{3-5}$ rapidly quenched ribbons,${ }^{6-8}$ mechanically milled powders, ${ }^{9-12}$ and self-assembled and core-shell nanoparticle systems. ${ }^{13,14}$ But scaling these processes up to bulk materials has remained to be a challenge. Traditional processing techniques such as casting and sintering are ill-suited for producing nanostructured bulk materials since long anneals at high temperatures inevitably lead to excessive grain growth. Nontraditional approaches to producing fully dense bulk nanocomposite magnets by warm compaction of $\mathrm{FePt}-\mathrm{Fe}_{3} \mathrm{O}_{4}$ nanoparticles (particle size of $\left.4-6 \mathrm{~nm}\right)^{15}$ and by shock compaction of nanoscale granular $\mathrm{Nd}-\mathrm{Fe}$ granular ribbons ${ }^{16}$ have achieved grain size control in the nanocomposite systems. In this report, we extend the warmcompaction process of severely deformed powders to fabrication of Sm-Co based bulk nanocomposite magnets with large energy product enhancement.

The raw materials of the nanocomposites were prepared by high-energy ball milling of the hard magnetic phase particles (commercially available $\mathrm{SmCo}_{5}$ or $\mathrm{Sm}_{2} \mathrm{Co}_{7}$ or $\mathrm{Sm}_{2} \mathrm{Co}_{17}$ ) and the soft magnetic phase particles (commercially available $\alpha-\mathrm{Fe})$ in micrometer size $(1-20 \mu \mathrm{m})$. Followed by warm compaction of the milled mixtures at different temperatures (room temperature to $700{ }^{\circ} \mathrm{C}$ ) under a pressure of $\sim 2.5 \mathrm{GPa}$, fully dense bulk samples were obtained. The ball milling process has two following purposes: to mix the two phases and to conduct severe plastic defor-

${ }^{\text {a)} E l e c t r o n i c ~ m a i l: ~ p l i u @ u t a . e d u . ~}$ mation of the micron sized particles to form composites with desired nanoscale morphology.

The effect of milling time was monitored by microscopic observations and magnetic measurements (Fig. 1). With increasing time, grain size was dramatically reduced and the energy filtered transmission electron microscopy (EFTEM) showed an unusual morphology of the soft magnetic phase. The initially equiaxed $\alpha$-Fe grains in microns became narrow and elongated with increasing milling time [Figs. 1(a) and 1(b)]. With further milling, the nanoscale $\alpha$-Fe strips began to break up into isolated equiaxed nanoscale grains [Figs. 1 (c) and 1(d)]. After $\sim 4 \mathrm{~h}$ of milling, homogeneously distributed magnetically soft phase particles were embedded in the hard-phase matrix with sizes smaller than $15 \mathrm{~nm}$. This

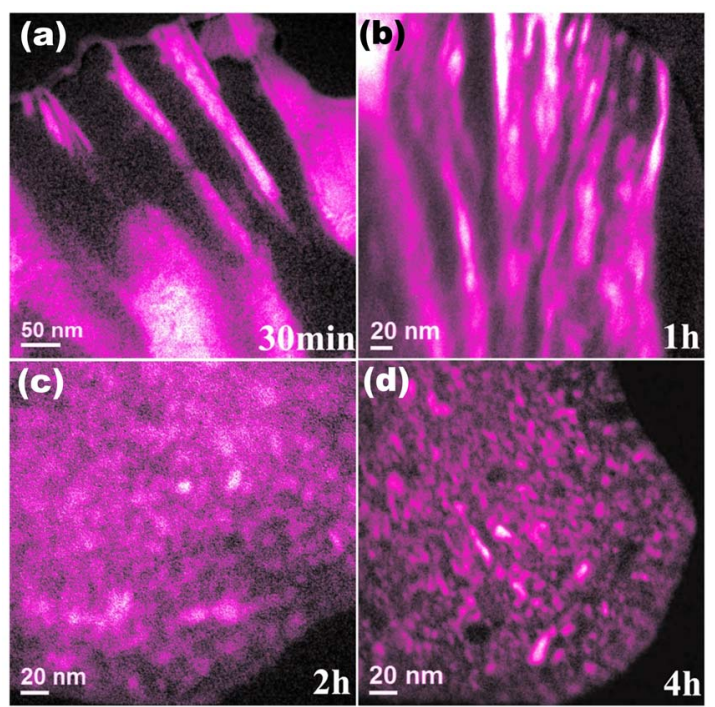

FIG. 1. (Color online) Morphology evolution during the deformation. Fe strips (dark contrast) can be observed in samples milled for more than 30 min; EFTEM showing Fe map of the samples milled for (a) $30 \mathrm{~min}$, (b) $1 \mathrm{~h}$, (c) $2 \mathrm{~h}$, and (d) $4 \mathrm{~h}$, respectively. High Fe regions have bright contrast. The Fe strips become thinner with increasing milling time. 


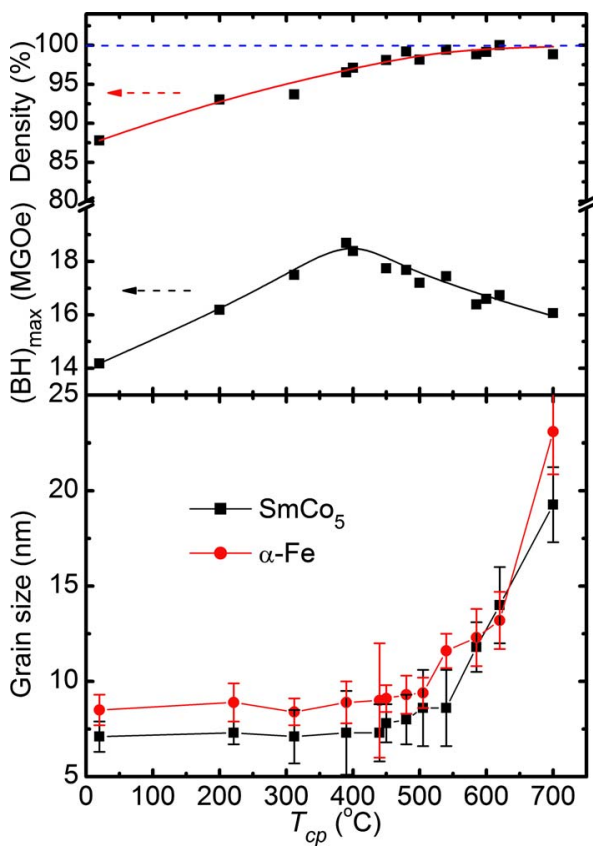

FIG. 2. (Color online) Dependence of density, average grain size and $(\mathrm{BH})_{\max }$ of the $\mathrm{SmCo}_{5} / \mathrm{Fe}$ bulk nanocomposite magnets on compaction temperature.

phenomenon induced by the severe plastic deformation can be attributed to the brittle-ductile two-phase deformation behavior. ${ }^{17,18}$ The magnetically hard/soft composite system is also a mechanically brittle/ductile system. The presence of the narrowed and elongated $\alpha$-Fe phase in the $\mathrm{SmCo} / \mathrm{Fe}$ system is in accord with previous observations by Kiparisov et $a l .{ }^{17}$ who showed that the ductile phase will undergo more plastic deformation than the brittle phase until it work- hardens. Meanwhile, another study by Chen $e t$ al. ${ }^{18}$ shows that plastic deformation can change a striped morphology of a composite into an equiaxed morphology by "necking" and breaking the strips. It is interesting to see that these two processes indeed take place in sequence in the $\mathrm{SmCo} / \mathrm{Fe}$ hard/soft composites, leading to nanoscaling of the soft phase and the formation of nanocomposites with desired nanoscale morphology.

Simulations have shown that the size of the soft magnetic phase in the exchange-coupled composites must be smaller than a critical dimension in a nanometer scale determined by the hard-phase domain wall thickness and the soft-phase magnetic properties. ${ }^{1,2,19,20}$ This critical dimension is the upper limit for all the soft-phase grains (not an average of the distribution). Our calculation gave the critical dimension of $\sim 20 \mathrm{~nm}$ for the Fe-based soft phase in the $\mathrm{SmCo}_{5}$ matrix. $^{20}$ Without enough refining of the morphology through ball milling, the $\alpha$-Fe particle size was larger than $20 \mathrm{~nm}$. From the demagnetization curves of the compacted and optimally annealed $\mathrm{SmCo}_{5}+20$ wt $\% \mathrm{Fe}$ samples milled for different times, it can be clearly seen that the curves for samples milled less than $2 \mathrm{~h}$ have a kink indicative of a decoupled two-phase magnet due to soft-phase grains that are larger than $20 \mathrm{~nm}^{21}$ Optimal milling time for the best energy product was $4 \mathrm{~h}$ which exhibited uniform nanoscale morphology.

Not only can the soft-phase distribution have an effect on the energy product but the compaction processing also plays an essential role in defining the bulk nanocomposite magnet performance. Warm compaction of the nanocomposite magnets was performed at temperatures from 20 to $700{ }^{\circ} \mathrm{C}$, with the processing details described in our previous report. ${ }^{15}$ Figure 2 shows the density, grain size, and energy-

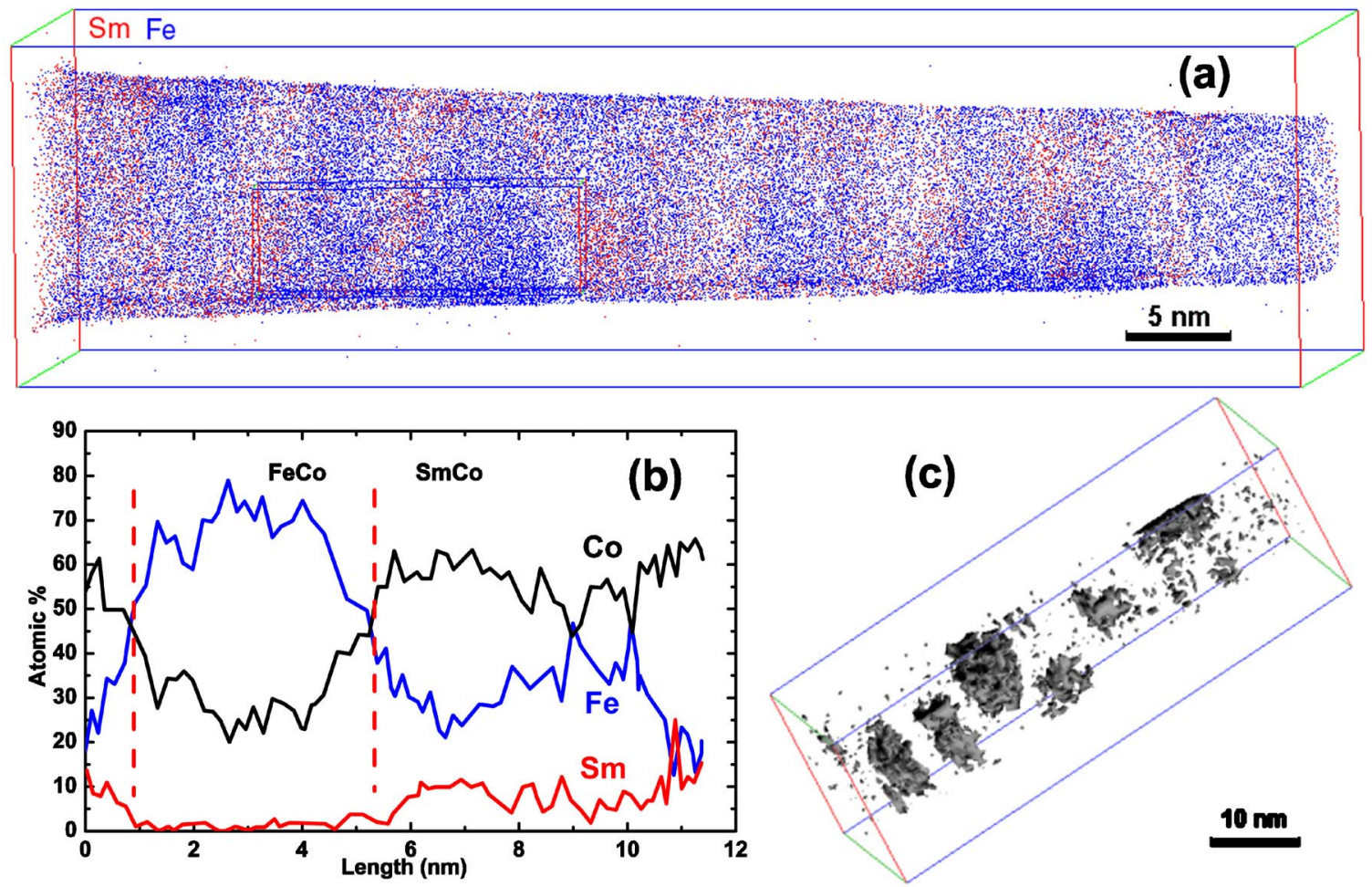

FIG. 3. (Color online) The 3D atom probe analysis for the $\mathrm{SmCo}_{5}+20 \mathrm{wt} \% \mathrm{Fe}$ nanocomposite bulk magnet. (a) 3D atom map of $\mathrm{Sm}$ and Fe, (b) composition profiles across the FeCo phase [box size $=4 \times 3 \times 12 \mathrm{~nm}^{3}$, shown in (a)], and (c) $60 \%$ Fe isoconcentration surface. Multimedia 1: 3D isoconcentration surface of iron [see (c)]. Multimedia 2: 3D atom map of Sm and Fe [see (c)] (enhanced online). [URL: http://dx.doi.org/10.1063/1.3358390.1]; [URL: http://dx.doi.org/10.1063/1.3358390.2]. 
product dependence on the compaction temperature for the $\mathrm{SmCo}_{5}$-based nanocomposite system. It is seen that the highest energy product can be achieved at temperatures around $400{ }^{\circ} \mathrm{C}$, based on the controlled grain size and the high density.

It has been observed that pressing time has also significant effect on the properties even in case no grain size change was detected. Longer holding time results in a higher energy product by improving the coercivity and the squareness of the hysteresis loops. The reason for this improvement can be attributed to the recent finding that interphase exchange coupling favors interdiffused interfaces (graded interface). ${ }^{22,23}$ To characterize the elemental composition in the bulk nanocomposite magnets, three-dimensional (3D) atom probe analysis has been conducted for a $\mathrm{SmCo}_{5}$ +20 wt $\%$ Fe nanocomposite bulk magnet. A 3D atom map of $\mathrm{Sm}$ and $\mathrm{Fe}$ is shown in Fig. 3. It is revealed that Fe-rich regions containing about $74 \pm 2$ at. $\% \mathrm{Fe}$ and $26 \pm 1$ at. \% Co distribute homogeneously in the sample. They were formed from those initially pure $\alpha$-Fe phase particles. The wavelike composition profile of the soft phase (graded interface), resulted from the interdiffusion during the processing, is consistent with the analysis given above. The interdiffusion has also led to increased magnetization in soft magnetic phase because a FeCo phase can have higher magnetization than pure Fe.

Based on the above observation and understanding, a soft phase with modified composition was added to the composite which has led to further improved energy products. The $(\mathrm{BH})_{\max }$ value for a $\mathrm{SmCo}_{5}+25$ wt $\% \mathrm{Fe}_{65} \mathrm{Co}_{35}$ nanocomposite is $19.2 \mathrm{MGOe}$, which is high in comparison to the nanocomposite with pure $\mathrm{Fe}$ addition (18.3 MGOe) and to the single-phase counterpart (9.0 MGOe, close to the theoretical value for $\mathrm{SmCo}_{5}$ phase). High temperature properties have been measured up to $1000 \mathrm{~K}$ and it has been found that at $573 \mathrm{~K}, 60 \%$ of the energy product value can be retained, which makes this type of materials suitable for high temperature applications. The details will be given elsewhere.

Similar investigations have also been carried out for the $\mathrm{Sm}_{2} \mathrm{Co}_{7}$-based nanocomposite systems. A more favorable situation has been found in the $\mathrm{Sm}_{2} \mathrm{Co}_{7}$-based systems where the energy product peaks at 17.0 MGOe when 35 wt \% Fe addition was made, which is significantly higher than that of the single-phase counterpart ( $\sim 4$ MGOe, close to the theoretical value for $\mathrm{Sm}_{2} \mathrm{Co}_{7}$ compound). This remarkable energy product enhancement can be attributed to the fact that $\mathrm{Sm}_{2} \mathrm{Co}_{7}$ has relatively low magnetocrystalline anisotropy compared to $\mathrm{SmCo}_{5}$. There is an anticorrelation between the hard-phase anisotropy constant and the critical soft-phase dimension. ${ }^{1}$ Thus the $\mathrm{Sm}_{2} \mathrm{Co}_{7}$-based composites have a larger critical soft-phase dimension and are expected to "tolerate" more soft-phase and soft-phase agglomeration. Our TEM observations have confirmed this phenomenon.

In conclusion, bulk $\mathrm{SmCo} / \mathrm{FeCo}$ hard/soft nanocomposite magnets have been fabricated using the enabling technology based on severe plastic deformation coupled with warm compaction. The substantial soft-phase fraction, well con- trolled grain size and modified graded interface have resulted in large energy product enhancement over the base singlephase counterparts in the isotropic nanocomposite systems. A severe-plastic-deformation-induced size reduction mechanism has been discovered in the magnetically and mechanically hard/soft composite systems, which can be applied to fabricating other types of nanocomposite materials as well.

This work was supported in part by the U.S. Department of Defense, Office of Naval Research/MURI Project under Grant No. N00014-05-1-0497 and by the University of Texas-Arlington. Work at the Ames laboratory was supported in part by the U.S. Department of Energy, Office of Basic Energy Science, under Contract No. DE-AC02-07CH11358. X.X. thanks Dr. Sergey Rubanov of University of Melbourne for his assistance in the atom probe investigation.

${ }^{1}$ E. F. Kneller and R. Hawig, IEEE Trans. Magn. 27, 3588 (1991).

${ }^{2}$ R. Skomski and J. M. D. Coey, Phys. Rev. B 48, 15812 (1993).

${ }^{3}$ J. P. Liu, C. P. Luo, Y. Liu, and D. J. Sellmyer, Appl. Phys. Lett. 72, 483 (1998).

${ }^{4}$ W. Liu, Z.-D. Zhang, J. P. Liu, L. J. Chen, L. L. He, Y. Liu, X. K. Sun, and D. J. Sellmyer, Adv. Mater. (Weinheim, Ger.) 14, 1832 (2002).

${ }^{5}$ J. Zhang, Y. K. Takahashi, R. Gopalan, and K. Hono, Appl. Phys. Lett. 86, 122509 (2005).

${ }^{6}$ A. Manaf, R. A. Buckley, and H. A. Davis, J. Magn. Magn. Mater. 128, 302 (1993).

${ }^{7}$ L. Withanawasam, G. C. Hadjipanayis, and R. F. Krause, J. Appl. Phys. 75, 6646 (1994).

${ }^{8}$ X. Y. Zhang, Y. Guan, L. Yang, and J. W. Zhang, Appl. Phys. Lett. 79, $2426(2001)$

${ }^{9}$ J. Ding, P. G. McCormick, and R. Street, J. Magn. Magn. Mater. 124, 1 (1993).

${ }^{10}$ K. O'Donnell, C. Kuhrt, and J. M. D. Coey, J. Appl. Phys. 76, 7068 (1994).

${ }^{11}$ Z. D. Zhang, W. Liu, X. K. Sun, X. Zhao, Q. Xiao, Y. Sui, and T. Zhao, J. Magn. Magn. Mater. 184, 101 (1998).

${ }^{12}$ J. Zhang, S. Y. Zhang, H. W. Zhang, B. G. Shen, and B. H. Li, J. Appl. Phys. 89, 2857 (2001).

${ }^{13}$ H. Zeng, J. Li, J. P. Liu, Z. L. Wang, and S. H. Sun, Nature (London) 420, 395 (2002).

${ }^{14}$ V. Nandwana, G. S. Chaubey, K. Yano, C. B. Rong, and J. P. Liu, J. Appl. Phys. 105, 014303 (2009).

${ }^{15}$ C. B. Rong, V. Nandwana, N. Poudyal, J. P. Liu, M. E. Kozlov, R. H Baughman, Y. Ding, and Z. L. Wang, J. Appl. Phys. 102, 023908 (2007).

${ }^{16}$ K. H. Chen, Z. Q. Jin, J. Li, G. Kennedy, H. Zeng, S.-F. Cheng, Z. L. Wang, N. N. Thadhani, and J. P. Liu, J. Appl. Phys. 96, 1276 (2004).

${ }^{17}$ S. S. Kiparisov, I. A. Kiyanskii, and V. E. Perel'man, Powder Metall. Met. Ceram. 26, 899 (1987).

${ }^{18}$ I.-W. Chen, E. J. Winn, and M. Menon, Mater. Sci. Eng., A 317, 226 (2001).

${ }^{19}$ Z. S. Shan, J. P. Liu, V. M. Chkka, H. Zeng, and J. S. Jiang, IEEE Trans. Magn. 38, 2907 (2002)

${ }^{20}$ Z. J. Guo, J. S. Jiang, J. E. Pearson, S. D. Bader, and J. P. Liu, Appl. Phys. Lett. 81, 2029 (2002).

${ }^{21}$ J. P. Liu, in Nanoscale Magnetic Materials and Applications, edited by J. P. Liu, E. Fullerton, O. Gutfleisch, and D. J. Sellmyer (Springer, New York, 2009), p. 316

${ }^{22}$ J. S. Jiang, J. E. Pearson, Z. Y. Liu, B. Kabius, S. Trasobares, D. J. Miller, S. D. Bader, D. R. Lee, D. Haskel, G. Srajer, and J. P. Liu, Appl. Phys. Lett. 85, 5293 (2004).

${ }^{23}$ Y. Choi, J. S. Jiang, Y. Ding, R. A. Rosenberg, J. E. Pearson, S. D. Bader, A. Zambano, M. Murakami, I. Takeuchi, Z. L. Wang, and J. P. Liu, Phys. Rev. B 75, 104432 (2007). 